\title{
An improved Reynolds technique for approximate solution of linear
}

\author{
stochastic differential equations
}

\author{
J. STAHLBERG \\ Astrophysikalisches Institut Potsdam \\ Rosa Luxemburg Str.17a, D-O-1590 Potsdam, Germany
}

Our starting point is a formal linear stochastic differential equation of first order (higher order equations can be transformed to systems of these)

$$
\frac{d I(x, \omega)}{d x}=a(x, \omega) I(x, \omega)-W(x, \omega ; I)
$$

where $I, a, W$ are stochastic functions $I=\bar{I}+I^{\prime}, a=\bar{a}+a^{\prime}, W=\bar{W}+W^{\prime}$, with $\langle I\rangle=\bar{I},\left\langle I^{\prime}\right\rangle=0$, and analogously for $a$ and $W . I, a$, and $W$ are allowed to depend on the element $\omega$ of a set $\Omega$ in which a probability measure is defined in the usual way (see e.g. Doob, 1953; de Witt-Morette, 1981). To get a solution of eq.(1) for the mean intensity $\bar{I}$ we treat the problem according the Reynolds averaging technique in the usual manner : The stochastic equation is changed into an infinte hierarchical system of equations for the correlations. At first we take the mean of eq.(1)

$$
\frac{d<I(x)>}{d x}=\bar{a}(x) \bar{I}(x)+<a^{\prime}(x) I(x)>-\bar{W}(x) .
$$

Multiplying eq.(1) with $a^{\prime}\left(x^{\prime}\right)$ resp. with $a^{\prime}\left(x^{\prime}\right) a^{\prime}\left(x^{\prime \prime}\right)$ and so on we get by taking the mean

$$
\begin{gathered}
\frac{d}{d x}<a^{\prime}\left(x^{\prime}\right) I(x)>=<a^{\prime}\left(x^{\prime}\right)(a(x) I(x)-W(x))> \\
\ldots \\
\frac{d}{d x}<a^{\prime}\left(x^{(n)}\right) \ldots a^{\prime}\left(x^{\prime}\right) I(x)>=<a^{\prime}\left(x^{(n)}\right) \ldots a^{\prime}\left(x^{\prime}\right)(a(x) I(x)-W(x))>
\end{gathered}
$$

where $x^{(n)}$ is a $n$-dashed $\mathrm{x}$ (dependence on $\omega$ is omitted). The eqs.(2) and (3) form an infinite hierarchical system of differential equations. A (calculable) solution can be obtained by truncating the infinite system. A simple cut off, often used in physics, consists in neglecting higher order correlations $(n>2)$. Thus we get for $n=2$ :

$$
<a^{\prime}\left(x^{\prime}\right) a^{\prime}(x) I(x)>=<a^{\prime}\left(x^{\prime}\right) a^{\prime}(x)>\bar{I}(x)
$$

$\left(<a^{\prime} a^{\prime} I^{\prime}>=0\right.$ ). For small deviations from the solution of the completely random (uncorrelated) case, the cut off according to eq.(4) is a valid approximation. However, especially for larger fluctuations far away from the totally random case the cut off according to eq.(4) becomes incorrect: It completely neglect higher order correlations. To take into account these higher order correlations via 2- point correlations (as does e.g. a Markow process) resp. via n-point correlations, we approximate the left side of eq.(5) in such a way that the two known limiting cases of 
the stochastic process, the solution of the completely random case and the solution $\left\langle\mathbf{I}_{\infty}\right\rangle$ of a totally correlated stochastic process, become exactly included:

$$
\left\langle\mathbf{a}^{\prime}\left(\mathbf{x}^{\prime}\right) \mathbf{a}^{\prime}(\mathbf{x}) \mathbf{I}(\mathbf{x})\right\rangle=\left\langle\mathbf{a}^{\prime}\left(\mathbf{x}^{\prime}\right) \mathbf{a}^{\prime}(\mathbf{x}) \mathbf{I}_{\infty}(\mathbf{x})\right\rangle,
$$

where $\mathbf{I}_{\infty}$ can be obtained by including $\omega$ in the nonstochastic(!) solution $I_{o}$ of eq.(1): $I_{o}(x) \rightarrow I_{o}(x, \omega)($ solution of eq.(1)in the totally correlated case only!) $\equiv$ $I_{\infty}$. In the $\mathrm{n}$-th order we get the generalised form

$$
<\mathbf{a}^{\prime}\left(\mathbf{x}^{(\mathbf{n})}\right) \ldots \mathbf{a}^{\prime}(\mathbf{x}) \mathbf{I}(\mathbf{x})>=<\mathbf{a}^{\prime}\left(\mathbf{x}^{(\mathbf{n})}\right) \ldots \mathbf{a}^{\prime}(\mathbf{x}) \mathbf{I}_{\infty}(\mathbf{x})>.
$$

The eqs.(5) are the simplest ansatz to fulfil the above made conditions. A more complex ansatz could give better results.

Cutting off the system of the eqs.(2) and (3) according to eq.(5b) we get

$$
\begin{gathered}
\bar{I}(x)=\mathbf{\Lambda}<W\left(x^{\prime}\right)>-\boldsymbol{\Lambda}^{2}<a^{\prime}\left(x^{\prime}\right) W\left(x^{\prime \prime}\right)>+\ldots+-\mathbf{\Lambda}^{n}<a^{\prime}\left(x^{\prime}\right) \ldots a^{\prime}\left(x^{(n-1)}\right) \\
\left(W\left(x^{(n)}\right)-a^{\prime}\left(x^{(n)}\right) I_{\infty}\left(x^{(n)}\right)\right)>+\Delta_{n}
\end{gathered}
$$

with

$$
\begin{gathered}
\boldsymbol{\Lambda} X(x)=\exp \left(\int_{0}^{x} \bar{a}\left(x^{\prime}\right) d x^{\prime}\right) \int_{x}^{\infty} d x^{\prime} \exp \left(-\int_{0}^{x^{\prime}} \bar{a}\left(x^{*}\right) d x^{*}\right) X\left(x^{\prime}\right) \\
\mathbf{\Lambda}^{2} X\left(x^{\prime}\right)=\boldsymbol{\Lambda}\left(\exp \left(\int_{0}^{x^{\prime}} \bar{a}\left(x^{*}\right) d x^{*}\right) \int_{x^{\prime}}^{\infty} d x^{\prime \prime} \exp \left(-\int_{0}^{x^{\prime \prime}} a\left(x^{\$}\right) d x^{\$}\right) X\left(x^{\prime \prime}\right)\right.
\end{gathered}
$$

and the error term $\left.\Delta_{n}=\Lambda^{n}<a^{\prime}\left(x^{\prime}\right) \ldots a^{\prime}\left(x^{(n)}\right)\left(I-I_{\infty}\right)\right\rangle$.

If for a special stochastic process any $\Delta_{n}=0$ we have found an exact solution. In general there are no closed systems of equations and a $\Delta_{n}$ is assumed to be sufficiently small to cut off the infinite system. From eq.(6) some special cases follow:

a) $\Delta_{1}=0$ : On the one hand it is: $\bar{I}=\boldsymbol{\Lambda}\left(\bar{W}-<a^{\prime} I_{\infty}>\right)$, a totally correlated stochastic process and on the other hand $\bar{I}=\boldsymbol{\Lambda} \bar{W}$, a completely randomly stochastic process.

b) $\Delta_{2}=0: \bar{I}(x)=\Lambda \bar{W}\left(x^{\prime}\right)-\Lambda^{2}<a^{\prime}\left(x^{\prime}\right)\left(W\left(x^{\prime \prime}\right)-a^{\prime}\left(x^{\prime \prime}\right) I_{\infty}\left(x^{\prime \prime}\right)>\right.$.

Eq.(7) is (by including of finite correlations) the simplest solution of eq.(1) in the frame of the proposed cut off. It is comparable with solutions in the frame of a Markov process (MP) but is not restricted to special velocity fields as does the MP. Eq.(6) is not only applicable to small perturbations caused by a random process. By taking into account even higher order momentum the accuracy increases like a power law : $D^{n} / n$ !, where $D=\left(f\left(v_{m i}\right)-f\left(v_{m a}\right)\right) / f\left(v_{o}\right)$. If $D>1$ then the validity decreases up to a limited $\mathrm{n}$ and then increases! For $n \geq D$ this series expansion is absolutly convergent. Eq.(7) as well as eq.(6) become exact for completely random and totally correlated stochastic processes.

\section{References}

De Witt-Morette,C., Elworthy, K.D.: 1981, Stochastic Diffential Equations, Proceedings of the "5-Tage-Kurs", Bielefeld, University,.

Doob,J.L.: 1953, Stochastic Processes, Wiley, New York, . 